\title{
Long-range rapidity correlations between mean transverse momenta in the model with string fusion
}

\author{
Vladimir Vechernin ${ }^{1, \star}$ \\ ${ }^{1}$ Saint Petersburg State University
}

\begin{abstract}
The long-range correlation between mean-event transverse momenta, being robust against the volume fluctuations and the details of the centrality determination, enables to obtain the signatures of string fusion at the initial stage of hadronic interaction in relativistic heavy ion collisions. The dependence of the correlation strength between mean-event transverse momenta on the collision centrality and initial energy is analyzed in a simple model with quark-gluon string fusion on the transverse lattice. It is shown that above RHIC energy the dependence reveals the decline of the correlation coefficient for most central collisions, reflecting the attenuation of color field fluctuations due to the string fusion at large string density. It is also found that contrary to the correlation between transverse momenta of single particles the strength of the correlation between mean-event transverse momenta of particles in two separated rapidity intervals is not decreasing with the total number of produced strings, remaining significant even in the case of $\mathrm{Pb}-\mathrm{Pb}$ collisions, in which the total number of strings can reach several thousand.
\end{abstract}

\section{Introduction}

The study of the correlations between observables in two separated rapidity windows (the so called long-range forward-backward correlations) has been proposed [1] as a signature of the string fusion and percolation phenomenon [2-5], which is one of the collectivity effects in ultrarelativistic heavy ion collisions. Later it was realized [6-11] that the investigations of the forward-backward correlations between intensive observables, such e.g. as mean-event transverse momenta, enable to obtain more clear signal about the initial stage of hadronic interaction, including the process of string fusion, compared to usual forward-backward multiplicity correlations.

In the present work, as an example, we consider the correlation between mean-event transverse momenta of charged particles in separated rapidity intervals $[8,10,11]$ :

$$
p_{t F}=\frac{1}{n_{F}} \sum_{i=1}^{n_{F}}\left|\mathbf{p}_{t F}^{i}\right| \quad p_{t B}=\frac{1}{n_{B}} \sum_{i=1}^{n_{B}}\left|\mathbf{p}_{t B}^{i}\right|
$$

The calculations are fulfilled in a simple model with string fusion by introducing a lattice in transverse plane $[7,12]$. Previously, in the framework of the model we found the analytical asymptotics of the correlation coefficient, $b_{p_{t} p_{t}}$, between mean transverse momenta in separated rapidity intervals at large

^e-mail: v.vechernin@spbu.ru 
string density and fulfilled, using MC simulations, the calculation of the correlation coefficient as a function of string density $[10,11]$. In present paper we discuss the impact of the obtained results on the $b_{p_{t} p_{t}}$ correlation coefficient centrality dependence at RHIC and LHC energies.

\section{Forward-Backward Rapidity Correlations}

Usually the forward-backward (FB) rapidity correlation are studied as the correlation between two observables, $F$ and $B$, measured event-by-event in some rapidity intervals, $\delta \eta_{F}$ and $\delta \eta_{B}$, separated by some rapidity gap, $\eta_{\text {gap }}$. Numerically it can be described by the correlation function:

$$
\langle B\rangle_{F}=f(F),
$$

giving the dependence of the mean value of the variable $B$ on the variable $F$. Usually this dependence is approximated by a linear function

$$
\langle B\rangle_{F}=a+b_{F B} F,
$$

the so-called linear regression. In this case the strength of the correlation can be characterized by one number - the correlation coefficient $b_{F B}$, for which one can find the following expression:

$$
b_{F B}=\frac{\langle F B\rangle-\langle F\rangle\langle B\rangle}{\left\langle F^{2}\right\rangle-\langle F\rangle^{2}}=\frac{\operatorname{cov}(F, B)}{D_{F}}
$$

To reduce the influence of different widths of the rapidity windows, $\delta \eta_{F}$ and $\delta \eta_{B}$, on the correlation coefficient the analysis sometimes performs using the relative variables, $F /\langle F\rangle$ and $B /\langle B\rangle$. Clear that the correlation coefficient in this variables, $b_{F B}^{r e l}$, is simply connected with the $b_{F B}: b_{F B}^{r e l}=b_{F B}\langle F\rangle /\langle B\rangle$. In our analysis we will use only symmetric observation windows, $\delta \eta_{F}=\delta \eta_{B}$, in this case $b_{F B}^{r e l}=b_{F B}$ for a symmetric collision. Nevertheless note, that this does not eliminates the dependence of the correlation coefficient on the rapidity width of observation window (see e.g. [13]) what is important when one compares the results of different experiments.

Due to the locality of strong interaction in rapidity the FB correlation naturally divides into two parts: the short- and the long-range ones. The Short-Range Correlation (SRC) takes place only between particles produced from a same source (string). It goes to zero for large enough rapidity gaps, $\eta_{\text {gap }} \gtrsim 2$. The origin of the Long-Range Correlations (LRC) at large $\eta_{\text {gap }}$ is event-by-event variance in the number and properties of particle sources (cut pomerons [14], strings [13] and so on), formed in high energy hadronic interaction. In present paper we will consider only these LRC, as we are interested in the study of changing of source properties (the string properties due to their fusion).

Traditionally in the FB correlation analysis the multiplicities of charged particles with rapidities belonging to these FB windows were used as the observables, $F=n_{F}$ and $B=n_{B}$. Unfortunately the $n-n$ LRC is dominated mainly by the fluctuation in the number of initial sources (strings) - the so-called "volume" fluctuation, originating, for example, due to the fluctuation of the collision impact parameter. The influence of the other interesting effects, connected with fluctuation of source properties (e.g. due to possible string fusion processes), on this type of correlation is rather small [15]. The reason is the extensive nature of these observables.

More clear signal about a variation of sources properties we can expect for the LRC correlations involving intensive observables, e.g. the mean $p_{t}$, (1). The simple "volume" fluctuations do not lead to the correlation between the intensive observables and we need the fluctuation in "quality" of sources for its explanation. So we have to study the $p_{t}{ }^{-} p_{t}$ LR correlation with $F=p_{t F}$ and $B=p_{t B}$, (1), as FB observables, if we aim to discover the possible signatures of string fusion phenomenon. 


\section{The model}

The discrete version of string fusion model $[4,5]$ with a lattice in transverse plane was introduced in [12] and then was successfully exploited for a description of various phenomena in ultra relativistic nuclear collisions [7-11, 16-18]. In this approach one splits the impact parameter plane into $M$ cells with the area equal to the transverse area of single string, $\sigma_{s t r}$, and supposes the fusion of all strings with the centers in a given cell. Then each string configuration is characterized by the set of integers:

$$
C_{\eta}=\left\{\eta_{1}, \ldots, \eta_{M}\right\} \text {, }
$$

where $\eta_{i}$ is a number of initial strings fused in a given $i$-th cell . This leads to the splitting of the transverse area into domains with different, fluctuating values of color field within them. What is similar to the attempts to take into account the density variation in transverse plane in models based on the BFKL evolution [19] and on the CGC approach [20].

In present calculations we supposed that the $\eta_{i}$ in each cell fluctuates around the same mean value $\bar{\eta}$ with a scaled variance $\omega_{\eta}$. Then in accordance with the string fusion prescription $[4,5]$ the mean number of charged particles in forward and backward observation windows, produced from the fragmentation of fused strings in the $i$-th cell, is given by the expression

$$
\bar{n}_{i}^{F}=\mu_{F} \sqrt{\eta_{i}},
$$

and the same for $\bar{n}_{i}^{B}$, where $\mu_{F}$ is a mean number of particles produced from decay of a single string in the forward window. Because we study only LRC, we also suppose the independent fragmentation of the $i$-th fused string in forward and backward windows. So the corresponding numbers of charge particles $n_{i}^{F}$ and $n_{i}^{B}$ fluctuates independently event-by-event around the above mean values with some scaled variance $\omega_{\mu}$.

Finally, in accordance with the string fusion ideas $[4,5]$ we suppose that the transverse momentum distribution of particles, produced from the $\eta_{i}$ fused strings, has enlarged mean transverse momentum:

$$
\bar{p}\left(\eta_{i}\right)=p_{0} \sqrt[4]{\eta_{i}},
$$

where $p_{0}$ is a mean transverse momentum for particles produced from a decay of a single string. For popular transverse momentum distributions $\varphi(p)$ with one dimensional parameter $\tilde{p}$ (see table 1), due to dimensional reasons, we have $\tilde{p} \sim \sqrt[4]{\eta_{i}}$. We have also the following connection between the variance and the mean transverse momentum for particles produced from a decay of the fused string in the $i$-th cell:

$$
d_{p_{i}}\left(\eta_{i}\right)=\gamma \bar{p}^{2}\left(\eta_{i}\right) \text {, }
$$

where the dimensionless parameter $\gamma$ depends only on the shape of the distribution (see table 1).

So each event in the model is characterized by the following configuration

$$
C=\left\{C_{\eta}, C_{n}^{F}, C_{n}^{B}, C_{p}^{F}, C_{p}^{B}\right\},
$$

where

$$
\begin{gathered}
C_{n}^{F}=\left\{n_{1}^{F}, \ldots, n_{M}^{F}\right\}, \quad C_{n}^{B}=\left\{n_{1}^{B}, \ldots, n_{M}^{B}\right\}, \\
C_{p}^{F}=\left\{p_{1}^{1 F}, \ldots, p_{1}^{n_{1}^{F} F} ; \ldots ; p_{M}^{1 F}, \ldots, p_{M}^{n_{M}^{F} F}\right\}
\end{gathered}
$$

and the same for the $C_{p}^{B}$. Here $n_{i}^{F}$ and $n_{i}^{B}$ are the numbers of charged particles, produced in forward and backward windows from the fragmentation of fused string in $i$-th cell, and $p_{i}^{j F}$ and $p_{i}^{j B}$ are their 
Table 1. The values of the dimensionless parameter $\gamma$, see formula (8), for the popular transverse momentum distributions $\varphi(p)$, normalized as $\int \varphi(p) d^{2} p=1(m>4)$.

\begin{tabular}{ll}
\hline$\varphi(p)$ & $\gamma$ \\
\hline$\sim \exp \left(-p^{2} / \tilde{p}^{2}\right)$ & $\frac{4-\pi}{\pi}$ \\
$\sim \exp (-p / \tilde{p})$ & $\frac{1}{2}$ \\
$\sim 1 /(1+p / \tilde{p})^{m}$ & $\frac{m-1}{2(m-4)}$
\end{tabular}

transverse momenta. Then, for the physical observables, defined in Sect. 1, we have

$$
p_{t F}=\frac{1}{n_{F}} \sum_{i=1}^{M} \sum_{j=1}^{n_{i}^{F}} p_{i}^{j F}, \quad n_{F}=\sum_{i=1}^{M} n_{i}^{F}
$$

and the same for the $p_{t B}$ and $n_{B}$. To calculate the correlation function (2) and the correlation coefficient (4) we have to average over these configurations with a proper weights (see [11,21] for details).

\section{The asymptotic of the correlation coefficient}

In the framework of the model one can find the explicit analytical asymptotic of the LR correlation coefficient between mean-event transverse momenta [11] at large uniform mean string density $\bar{\eta} / \sigma_{\text {str }}$ with $\bar{\eta} \gg 1$ :

$$
b_{p_{t} p_{t}}=\frac{\omega_{\eta} \mu_{F}}{\omega_{\eta} \mu_{F}+16 \gamma \sqrt{\bar{\eta}}} .
$$

The dimensionless parameter $\bar{\eta}$ characterizes the mean degree of overlap of strings. In the derivation of the asymptotic the existence of two small parameters: $1 / M$ and $1 / \bar{\eta}$ in the model was used. One can estimate the typical value of $M$, as ratio of the total transverse interaction area $S$ to the area of a single string $\sigma_{s t r}=\pi r_{s t r}^{2}$ :

$$
M \sim S / \sigma_{s t r} \sim R_{A}^{2} / r_{s t r}^{2} .
$$

For $\mathrm{Pb}-\mathrm{Pb}$ collisions $R_{A}=7 \div 8 \mathrm{fm}, r_{\text {str }}=0.2 \div 0.3 \mathrm{fm}$, so in this case we have $M \sim 10^{3}$. The value of $\bar{\eta} \sim 10$ at LHC and $\bar{\eta} \sim 3$ at RHIC energies [10, 22] (as we'll see below the asymptotic (13) is a good approximation only at $\bar{\eta} \gtrsim 7 \div 8$ ).

The most interesting feature of the asymptotic is the decrease of the correlation coefficient $b_{p_{t} p_{t}}$ with growth of the string density. The physical reason of such behavior is the attenuation of color field fluctuations due to the string fusion processes at large string density.

Another important property of the asymptotic is the $M$-scaling. The expression (13) for the $b_{p_{t} p_{t}}$ depends only on the string density $\bar{\eta}$ and does not depend on the total number of cells, $M$, and hence on the total number of strings. Note that this nontrivial property takes place only for the correlation between mean event transverse momenta, defined above, (1), in contrast with the behavior of the $p_{t}-p_{t}$ correlation coefficient, $\beta_{p_{t} p_{t}}$, between transverse momenta of two single particles, defined as

$$
\beta_{p_{t} p_{t}} \equiv \frac{I_{2}\left(p_{t 1}, p_{t 2}\right)}{I\left(p_{t 1}\right) I\left(p_{t 2}\right)}-1
$$


where $I\left(p_{t 1}\right)$ and $I_{2}\left(p_{t 1}, p_{t 2}\right)$ are single and double inclusive cross sections. In [7] it was shown that this correlation coefficient decreases with the total number of sources (strings), and hence with $M$ :

$$
\beta_{p_{t} p_{t}} \sim 1 / N_{\text {sources }} \sim 1 / M
$$

So, its value becomes very small, e.g. for $\mathrm{Pb}-\mathrm{Pb}$ interactions at LHC energy, in which the number the strings is of order of few thousand $[10,22]$. The reason of the difference between these two types of $p_{t^{-}}$ $p_{t}$ correlation, characterized by $b_{p_{t} p_{t}}$ and $\beta_{p_{t} p_{t}}$, is that the mean $p_{t F}$ and $p_{t B}$, being intensive variables, stronger correlates with the value of string tension and better reflects the specific event transverse energy in observation rapidity windows $\delta \eta_{F}$ and $\delta \eta_{B}$, than the transverse momenta of single particles.

We see also that the asymptotic (13) depends only on the one combination of all model parameters, $\frac{\omega_{\eta} \mu_{F}}{\gamma \sqrt{\bar{\eta}}}$, what we'll refer on as $\frac{\omega_{\eta} \mu_{F}}{\gamma \sqrt{\bar{\eta}}}$ - scaling. Note also, that in contrast with the correlations between multiplicities $n_{F}$ and $n_{B}$ and between mean transverse momentum $p_{t B}$ and multiplicity $n_{F}$, considered in [12], there is no dependence of the $b_{p_{t} p_{t}}$ asymptotic on the parameter $\omega_{\mu}$. Recall that $\omega_{\mu}$ characterizes the fluctuations in the number of particles produced from a string.

Instead of the $\omega_{\mu}$ we have the dependence of the $b_{p_{t} p_{t}}$ asymptotic on the dimensionless parameter $\gamma$, which characterizes the transverse momentum distribution from one initial string (see table 1). Since for a distribution with one dimensional parameter it does not depends on a string fusion, then in this condition it can be evaluated from data, as $\gamma=\left\langle\left\langle p_{t}^{2}\right\rangle\right\rangle /\left\langle\left\langle p_{t}\right\rangle\right\rangle^{2}-1$, where the $\langle\langle\ldots\rangle\rangle$ means averaging over tracks from all events.

\section{Results of the MC simulations}

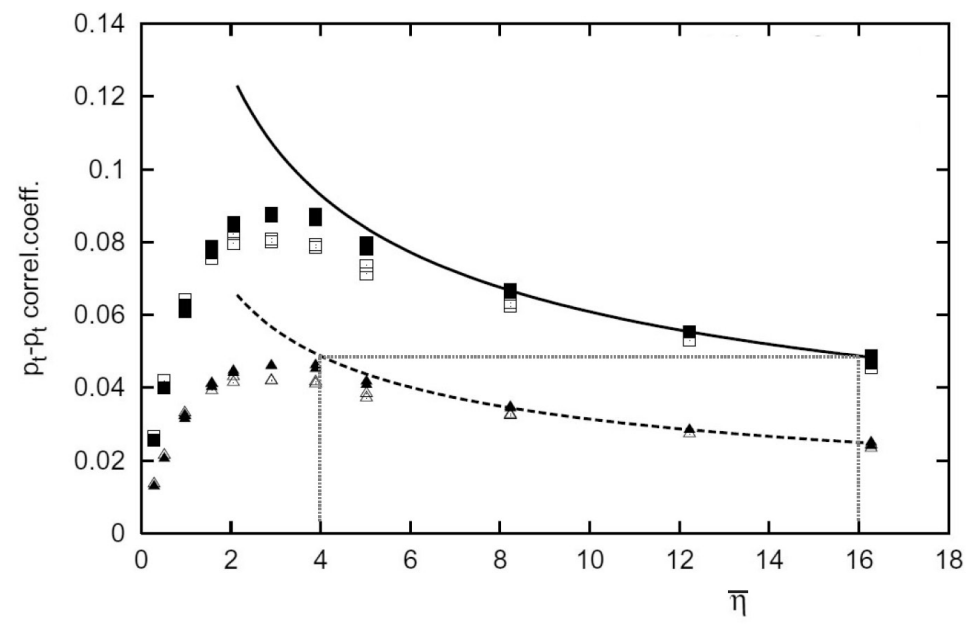

Figure 1. The $b_{p_{t} p_{t}}$ correlation coefficient (points), obtained by MC simulations in the model with string fusion on a transverse lattice with $M=45$ cells for $\mu_{F}=1(\triangle)$ and $\mu_{F}=2$ (口), as a function of mean string density $\bar{\eta}$. The filled points are calculated in the version with preliminary averaging of string density within lattice clusters (see $[10,23]$ for details). The curves - corresponding asymptotics calculated by the formula (13) at the parameter values: $\omega_{\eta}=\omega_{\mu}=1$ and $\gamma=0.6$. The dotted lines illustrate the $\mu_{F} / \sqrt{\eta}$-scaling for asymptotics (see text).

The MC simulations in the framework of this simple model with string fusion on a transverse lattice enable to calculate the value of the correlation coefficient $b_{p_{t} p_{t}}$, (4), for an arbitrary value and 


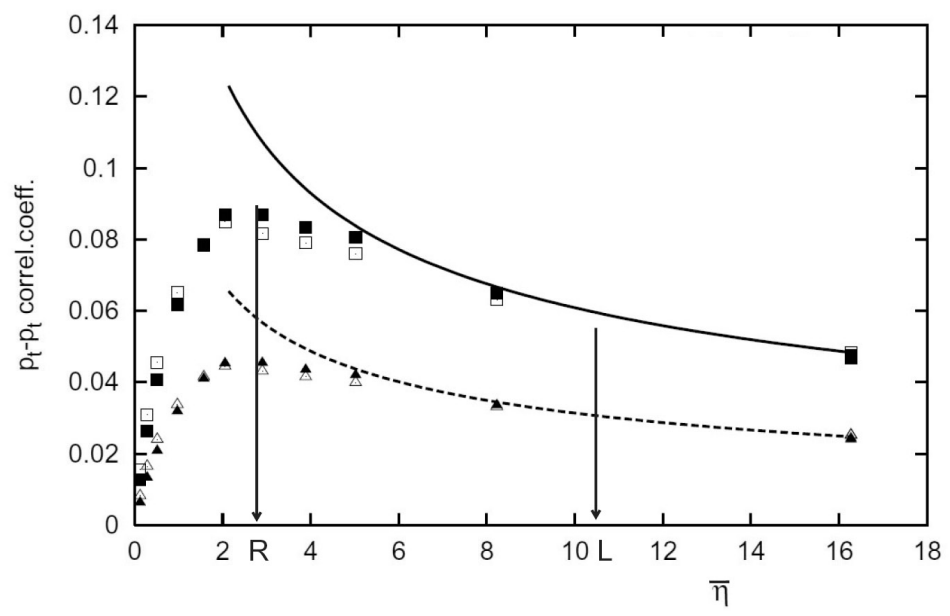

Figure 2. The same as in figure 1, but for a lattice with $M=450$ cells. The arrows show the string densities, $\mathrm{R}$ and $\mathrm{L}$, available correspondingly at RHIC and LHC [10, 22].

distribution of mean string density [10]. We restrict to the uniform string distribution over lattice with the mean density $\bar{\eta}$ and the poissonian fluctuations around this value $\left(\omega_{\eta}=1\right)$ in each cell. The results for the $b_{p_{t} p_{t}}$ correlation coefficient calculated on the base the MC simulation for $\mu_{F}=1$ and $\mu_{F}=2$ in the cases $M=45$ and $M=450$ are presented in figures 1 and 2 together with the corresponding asymptotics calculated by the formula (13).

First of all we see in these figures that the asymptotics well approximate the correlation coefficient $b_{p_{t} p_{t}}$ at values of $\bar{\eta}$ greater than $7 \div 8$. Comparing figures 1 and 2 we see also a good fulfillment of the $M$-scaling at any value of string density $\bar{\eta}$, and not only in the asymptotic region. (More thorough analysis [12] shows that the $M$-scaling is broken only in narrow region $\bar{\eta} \lesssim 1 / M$, when the total number of strings is small, $\bar{N}=M \bar{\eta} \sim 1$, so that the condition of formation at least one string in any inelastic collision starts to influence the results.)

In contrast to the $M$-scaling the $\mu_{F} / \sqrt{\eta}$-scaling is valid only in the asymptotic region $\bar{\eta} \gg 1$. One can see this from the dotted lines in figure 1. The lines illustrate that in this region the increase of the $\mu_{F}$ from 1 to 2 can be compensated by fourfold increase of the $\bar{\eta}$.

\section{Energy and centrality dependencies}

The general behavior of the $p_{t}-p_{t}$ correlation coefficient, $b_{p_{t} p_{t}}$, as a function of heavy ion collision centrality for RHIC and LHC energies is presented in figure 3. Qualitatively, it can be understood based on the dependency, shown in figure 2 , as follows.

The maximum string density, achieved for $0-10 \%$ of the most central Au-Au collisions at energy $200 \mathrm{GeV} /$ nucleon in RHIC, corresponds to the value $\bar{\eta}=2.88 \pm 0.09$ and the one for $0-5 \%$ of the most central $\mathrm{Pb}-\mathrm{Pb}$ collisions at energy $2.76 \mathrm{TeV} /$ nucleon in LHC reaches $\bar{\eta}=10.56 \pm 1.05$ [22]. So, depending on the collision centrality, it varies in the intervals $(0, \mathrm{R})$ for RHIC and $(0, \mathrm{~L})$ for LHC, shown in figure 2. In this figure we see that the increase of the string density $\bar{\eta}$ with centrality from 0 to R leads to the monotonic growth of the correlation coefficient, which at RHIC energies reaches the highest value in most central collisions, the left panel in figure 3. 


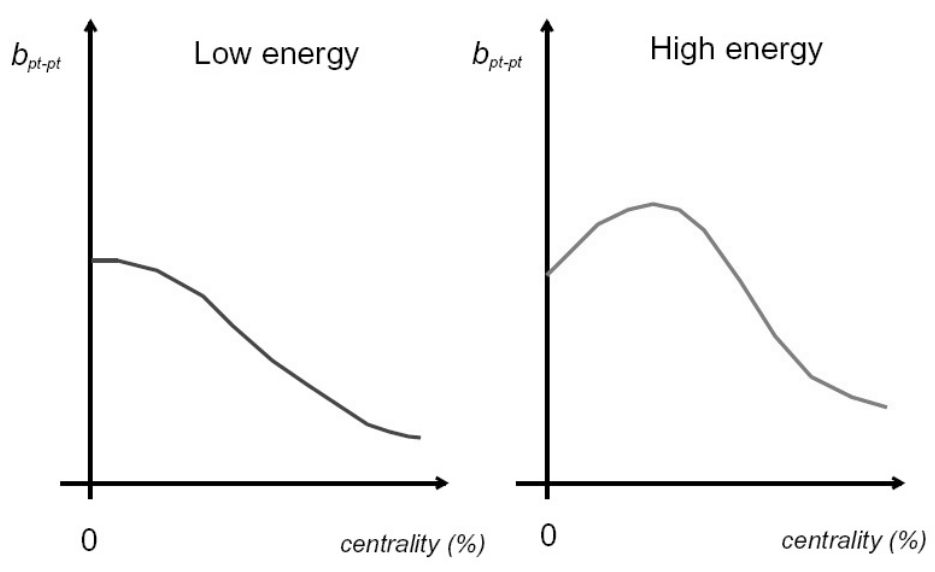

Figure 3. The general form of the dependence of $b_{p_{t} p_{t}}$ on the collision centrality at RHIC (left panel) and LHC (right panel), following from the existence of the $b_{p_{t} p_{t}}$ maximum at $\bar{\eta}=3 \div 4$ in figures 1 and 2 (see text).

At LHC energies, depending on the centrality, the string density $\bar{\eta}$ varies in the interval $(0, \mathrm{~L})$. In figure 2 we see that in this case the $p_{t}-p_{t}$ correlation coefficient, $b_{p_{t} p_{t}}$, reaches the highest value at some intermediate value of centrality, which corresponds to the string density $\bar{\eta} \simeq 3$. The further increase of $\bar{\eta}$ with centrality from 3 to L leads to the decrease of the correlation coefficient, in contrast with the situation at RHIC energies. So at LHC energies the $p_{t}-p_{t}$ correlation coefficient reaches the highest value in semicentral collisions at some intermediate value of centrality, see the right panel in figure 3 ,

This decrease of the correlation coefficient for the most central collisions at LHC energies corresponds to the decrease of the asymptotic (13) with the growth of $\bar{\eta}$, discussed in Sect. 4 . It is clear from the figure 2 that the RHIC data, corresponding to the $\bar{\eta}$ region $(0, \mathrm{R})$, can not be described by the asymptotic formula (13), which starts to work at $\bar{\eta} \gtrsim 7 \div 8$. This asymptotic, decreasing with string density, behavior is reached only for central heavy ion collisions at LHC energies. The model calculations show that the physical reason of this decrease is the attenuation of color field fluctuations due to the string fusion at large string density.

So the results show that the non-monotonic behaviour of $b_{p_{t} p_{t}}$ with centrality is achieved in heavy ion collisions at LHC, while at RHIC the string density is not enough to provide a decline of the correlation coefficient for most central collisions (see figure 3 ). This qualitative conclusion is confirmed by the results [24], obtained in more realistic dipole-based Monte Carlo string fusion model [25, 26].

\section{Summary and conclusions}

The $p_{t}$ - $p_{t}$ correlations between mean-event transverse momenta are studied in a simple model with quark-gluon string fusion on the transverse lattice. The dependence of the correlation coefficient, $b_{p_{t} p_{t}}$, characterizing the correlation strength, on the collision centrality is analyzed for different initial energies. It is shown that at LHC energy the dependence reveals the decrease of the correlation coefficient for most central collisions, reflecting the attenuation of color field fluctuations due to the 
string fusion at large string density, while at RHIC energy the string density is not yet enough to provide a decline of the correlation coefficient for most central collisions.

It is also found that contrary to the correlation between transverse momenta of single particles the strength of the correlation between mean-event transverse momenta of particles in two separated rapidity intervals is not decreasing with the total number of produced strings, remaining significant even in the case of $\mathrm{Pb}-\mathrm{Pb}$ collisions, in which the total number of strings can reach several thousand.

So the long-range correlation between mean-event transverse momenta, being robust against the volume fluctuations and the details of the centrality determination, enables to obtain the signatures of string fusion at the initial stage of hadronic interaction in relativistic heavy ion collisions at LHC energy.

\section{Acknowledgements}

The work was supported by the Saint-Petersburg State University grant 11.38 .242 .2015 and by the RFBR grant 15-02-02097-a.

\section{References}

[1] N.S. Amelin et al., Phys. Rev. Lett. 73, 2813 (1994)

[2] T.S. Biro, H.B. Nielsen, J. Knoll, Nucl. Phys. B 245, 449 (1984)

[3] A. Bialas, W. Czyz, Nucl. Phys. B 267, 242 (1986)

[4] M.A. Braun, C. Pajares, Phys. Lett. B 287, 154 (1992)

[5] M.A. Braun, C. Pajares, Nucl. Phys. B 390, 542 (1993)

[6] M.A. Braun, C. Pajares, Phys. Rev. Lett. 85, 4864 (2000)

[7] M.A. Braun, R.S. Kolevatov, C. Pajares, V.V. Vechernin, Eur. Phys. J. C 32, 535 (2004).

[8] ALICE collaboration et al., J. Phys. G 32, 1295 (2006), [Sect. 6.5.15]

[9] V.V. Vechernin, R.S. Kolevatov, Phys. Atom. Nucl. 70, 1797 (2007)

[10] V.V. Vechernin, R.S. Kolevatov, Phys. Atom. Nucl. 70, 1809 (2007)

[11] V.V. Vechernin, Theor. Math. Phys., 184, 1271 (2015)

[12] V.V. Vechernin, R.S. Kolevatov, Vestn. Peterb. Univ., Ser.4: Fiz. Khim., No.4, 11 (2004), arXiv: hep-ph/0305136

[13] V. Vechernin, Nucl. Phys. A 939, 21 (2015)

[14] A. Capella, A. Krzywicki, Phys. Rev. D 18, 4120 (1978)

[15] M.A. Braun, C. Pajares, Eur. Phys. J. C 16, 349 (2000)

[16] M.A. Braun, C. Pajares, Eur. Phys. J. C 71, 1558 (2011)

[17] M.A. Braun, C. Pajares, V.V. Vechernin, Nucl. Phys. A 906, 14 (2013)

[18] M.A. Braun, C. Pajares, V.V. Vechernin, Eur. Phys. J. A 51, 44 (2015)

[19] E. Levin, A.H. Rezaeian, Phys.Rev. D 84, 034031 (2011)

[20] A.Kovner., M. Lublinsky, Phys.Rev. D 83, 034017 (2011)

[21] V.V. Vechernin, Theor. Math. Phys. (2016) [in press]

[22] J. Dias de Deuset al., Eur. Phys. J. C 72, 2123 (2012)

[23] V. Vechernin, I. Lakomov, Proceedings of Science Baldin-ISHEPP-XXI, 072 (2012)

[24] V. Kovalenko, V. Vechernin, EPJ Web of Conferences 66, 04015 (2014)

[25] V.N. Kovalenko, Phys. Atom. Nucl. 76, 1189 (2013)

[26] V. Kovalenko, V. Vechernin, Proceedings of Science Baldin-ISHEPP-XXI, 077 (2012) 